\title{
Acquired von Willebrand Disease in a Child With Wilms' Tumor: Implications for Postoperative Regional Anesthesia
}

\author{
Habib Kedir, e, Dmitry Tumin ${ }^{\mathrm{b}, \mathrm{c}}$, Giorgio Veneziano ${ }^{\mathrm{c}, \mathrm{d}}$, Joseph D. Tobias ${ }^{\mathrm{b}, \mathrm{c}, \mathrm{d}}$
}

\begin{abstract}
Regional anesthetic techniques including epidural anesthesia are a safe and effective method of providing postoperative analgesia while avoiding the potential adverse physiologic effects of systemic opioids. However, clinical scenarios or co-morbid conditions such as abnormal coagulation function may preclude the use of neuraxial analgesia. We present a 2-year-old boy who presented for nephrectomy due to Wilms' tumor disease. Preoperative evaluation of coagulation function revealed an elevated partial thromboplastin related to acquired von Willebrand disease and therefore a wound catheter was used instead of epidural analgesia. Coagulation function guidelines for epidural anesthesia are reviewed and alternative techniques such as a wound catheter are discussed.
\end{abstract}

Keywords: Postoperative pain management; Pediatric anesthesia; Regional anesthesia; Wound catheter; Coagulation function; von Willebrand disease

\section{Introduction}

Wilms' tumor also known as nephroblastoma is the most common primary renal tumor in children, usually presenting between 6 months and 5 years of age $[1,2]$. The majority of patients present with an abdominal mass, abdominal pain, hypertension or hematuria. One recognized yet uncommon presentation is a disturbance in coagulation function related to acquired von Willebrand syndrome (AvWS) [3]. Bleeding episodes due to AvWS have been reported in patients affected by lymphoproliferative, myeloproliferative, cardiovascular,

Manuscript submitted August 13, 2018, accepted September 4, 2018

aThe Ohio State University School of Medicine, Columbus, OH, USA bDepartment of Pediatrics, The Ohio State University College of Medicine, Columbus, OH, USA

'Department of Anesthesiology and Pain Medicine, Nationwide Children's Hospital, Columbus, OH, USA

${ }^{\mathrm{d}}$ Department of Anesthesiology and Pain Medicine, The Ohio State University College of Medicine, Columbus, OH, USA

${ }^{\mathrm{e}}$ Corresponding Author: Habib Kedir, Department of Anesthesiology and Pain Medicine, Nationwide Children's Hospital, 700 Children's Drive, Columbus, OH 43205, USA. Email: habib.kedir@osumc.edu

doi: https://doi.org/10.14740/jmc3143w neoplasia, and autoimmune disorders [4]. AvWS in association with Wilms' tumor is characterized by bleeding related to platelet dysfunction and disturbances of coagulation function including an elevated partial thromboplastin time (PTT) [5].

Neuraxial techniques such as the epidural anesthesia provide effective analgesia following major surgical procedures including laparotomy or thoracotomy. However, neuraxial techniques including epidural anesthesia are contraindicated in patients with altered coagulation function due to the risk of epidural hematoma formation. We present a 2-year-old boy who presented for right radical nephrectomy due to Wilms' tumor disease. Preoperative evaluation revealed prolonged PTT and prolonged activated partial thromboplastin time (aPTT). Coagulation function guidelines for epidural anesthesia are reviewed and alternative techniques such as a wound catheter are presented.

\section{Case Report}

Institutional Review Board approval is not required at Nationwide Children's Hospital (Columbus, Ohio) for the presentation of single case reports. A 2-year-old, $12.1 \mathrm{~kg}$ toddler presented to his primary care provider with a chief complaint of cough and constipation. On physical exam, an abdominal mass was noted by his primary care physician and he was admitted for further evaluation. Computed tomography of the abdomen revealed a large retroperitoneal mass involving the right kidney, consistent with Wilms' tumor. His past medial history included a blocked tear duct and infection due to human metapneumovirus. He had no past surgical history except for an uncomplicated circumcision. His medications at the time of surgery were included enalapril and isradipine for blood pressure control. Preoperative blood pressure was $107 / 68 \mathrm{~mm}$ Hg. Physical exam was unremarkable except for a slightly distended abdomen. Preoperative laboratory results revealed hemoglobin $10.1 \mathrm{~g} / \mathrm{dL}$, hematocrit $32 \%$, and an elevated platelet count of 533,000/ $\mathrm{mm}^{3}$. Electrolytes, blood urea nitrogen, and creatinine were normal. Coagulation studies showed a prothrombin time $13.5 \mathrm{~s}$, a prolonged PTT of $43 \mathrm{~s}$. He was transported to the operating room and routine American Society of Anesthesiologists' monitors were placed. Anesthesia was induced with fentanyl and propofol followed by rocuronium to facilitate endotracheal intubation. Central venous and arterial cannulas were placed. Maintenance anesthesia consisted of sevoflurane in air and oxygen with intermittent doses of fentanyl. The tumor was resected without complica- 
tions. Surgical time was $4 \mathrm{~h}$ and $14 \mathrm{~min}$. A total of $375 \mathrm{~mL}$ of crystalloid was infused and $32 \mathrm{~mL}$ of urine was collected. Minimal blood loss was recorded for the procedure. Repeat coagulation studies revealed persistence of the prolonged aPTT (43 s), which contraindicated neuraxial epidural, so a wound catheter was placed during surgical closure. The wound catheter was inserted above closed parietal peritoneum and underneath the muscular fascia layer. At the completion of the case, the patient's trachea was extubated, and he was transferred to the post-anesthesia care unit. Postoperatively, a continuous local anesthetic wound infusion (CLAWI) was administered with ropivacaine $0.2 \%$ at $1.2 \mathrm{~mL} / \mathrm{h}$ for the initial 48 postoperative hours. Initial postoperative pain was assessed as 3 of 10 on the Face, Legs, Activity, Cry, Consolability (FLACC) pain scale. Additional analgesia was provided by intravenous acetaminophen $(10 \mathrm{mg} / \mathrm{kg}$ intravenously every $6 \mathrm{~h})$ and nursecontrolled analgesia with hydromorphone. Total consumption of intravenous hydromorphone was $4.04 \mathrm{mg}(0.33 \mathrm{mg} / \mathrm{kg})$ over the first 48 postoperative hours. FLACC scores were recorded every $2 \mathrm{~h}$ postoperatively with the majority being scored at 0 and a one-time maximum score of 5 for the initial 48 postoperative hours. The postoperative course was uncomplicated except for the need for ongoing medications for blood pressure control.

\section{Discussion}

Hemostasis is a complex process regulated by thrombogenic and anti-thrombogenic factors which balance coagulation and bleeding [6]. Primary hemostasis results from the interactions between platelets, the vessel wall, adhesive proteins, and coagulation factors leading to the formation of the initial platelet plug. Normally, platelets do not adhere to intact vascular endothelium. However, after a vascular injury, platelets attach to collagen and $\mathrm{VWF}$ in the subendothelial tissue, initiating the coagulation process $[6,7]$. A secondary function of vWF is to bind with plasma proteins such as factor VIII (FVIII) in its inactive state, thus preventing its rapid degradation by other proteases $[7,8]$. Deficiencies in genes encoding the vWF result in von Willebrand disease (vWD), the most common inherited disturbance of coagulation function [9]. Laboratory findings include both defective platelet function (aggregation) as well as a prolongation of the aPTT $[9,10]$.

AvWS is a rare bleeding disorder that usually occurs in individuals with no previous or family history of bleeding. Although it is similar to inherited vWD with regard to laboratory and clinical findings, the etiology of prolonged aPTT in AvWS involves a diverse group pathological conditions [4]. Since most AvWS patients synthesized normal vWF, the mechanisms responsible for producing dysfunctional coagulation in these patients include destruction of vWF by autoantibodies, absorption of $\mathrm{vWF}$ by malignant cell clones, and damage to high-molecular-weight (HMW) vWF multimers due to high shear stress [11]. One of the more common pediatric malignancies associated with AvWS is Wilms' tumor [12]. A retrospective evaluation of the coagulation profile of 190 children with Wilms' tumor revealed AvWS in eight for an incidence of approximately $4 \%$ [13].
These data would suggest that routine evaluation of coagulation function is indicated prior to neuraxial anesthesia in patients with Wilms' tumor. In patients without evidence of defects in coagulation function, Vandermuelen et al reported the incidence of epidural hematoma formation to be approximately 1: 220,000 after intrathecal anesthesia and 1: 150,000 after epidural anesthesia [14]. The American Society of Regional Anesthesia (ASRA) cautions against placing epidural catheters in patient with abnormal coagulation function due to increased risk for epidural hematoma [15]. Proceeding with neuraxial anesthesia in the presence of clinical anticoagulation or abnormal coagulation function can lead to severe, potentially devastating consequences from spinal hematoma formation. Sequelae may include requirement for surgical evacuation, residual lower extremity motor and sensory deficits, and permanent paraplegia.

Given such concerns, we chose to use Continuous Local Anesthetic Wound Infusion (CLAWI) for postoperative analgesia. The technique has emerged as attractive option in various clinical scenarios given its efficacy and ease of catheter placement [16-20]. Data in both adult and pediatric patients have demonstrated effective analgesia, decreased opioid requirements, and earlier return of bowel function. In a prospective trial, the continuous preperitoneal infusion of $0.2 \%$ ropivacaine at $10 \mathrm{~mL} / \mathrm{h}$ for $48 \mathrm{~h}$ following open colorectal resection reduced morphine consumption, improved pain relief, and accelerated postoperative recovery [16]. Similar results were reported following open hepatic surgery [19].

Efficacy has also been demonstrated in the pediatric population. In children with spina bifida undergoing major reconstruction of the lower urinary tract, a continuous infusion of $0.1 \%$ ropivacaine provided postoperative analgesia while reducing systemic opioid use and associated adverse effects [21]. In children undergoing laparotomy for complicated appendicitis, a multimodal regimen that included a CLAWI with $0.2 \%$ bupivacaine, paracetamol, and rescue intravenous morphine, served as a safe and effective technique for postoperative analgesia [17]. Anecdotally, we noted similar efficacy in our patient. Given the associated coagulation disturbance, a wound catheter was placed during surgical closure of the incision. During closure of the surgical wound, a multi-holed soaker catheter was inserted above the closed parietal peritoneum and underneath the muscular fascia layer, covering the full length of the wound. The infusion catheter was secured to the skin, the subcutaneous and skin layers closed followed by placement of sterile dressings. Our catheter placement technique is similar to previous studies that showed wound catheter inserted on the pre-peritoneal area or within the muscle fascia is associated with better pain control compared to the subcutaneous layer $[16,22]$. The CLAWI was used as part of a multimodal pain management strategy with intravenous acetaminophen and NCA-hydromorphone.

In conclusion, we describe the perioperative care of a 2-year-old toddler with Wilms' tumor and AvWS. Given the potential for coagulation defects as part of a paraneoplastic process, routine assessment of coagulation function is suggested prior to neuraxial anesthesia. Since epidural analgesia is contraindicated in these patients, multimodal analgesia that includes continuous wound infiltration with local anesthetics 
offers an effective alternative for postoperative management of pain. Although the analgesic effect of continuous wound infiltration of local anesthetics is inferior to that of epidural anesthesia, CLAWI has been shown to reduce opioid requirements, accelerate the return of bowel function, and improve the postoperative course. A multimodal pain management strategy that includes CLAWI is safe and effective technique for postoperative pain control in children undergoing open abdominal surgery.

\section{Funding Support}

Departmental funding.

\section{Financial Disclosure}

All authors disclose no financial support.

\section{Conflict of Interest}

The authors report no potential conflict of interest.

\section{References}

1. Breslow N, Olshan A, Beckwith JB, Green DM. Epidemiology of Wilms tumor. Med Pediatr Oncol. 1993;21(3):172-181.

2. Breslow NE, Beckwith JB, Perlman EJ, Reeve AE. Age distributions, birth weights, nephrogenic rests, and heterogeneity in the pathogenesis of Wilms tumor. Pediatr Blood Cancer. 2006;47(3):260-267.

3. Leung RS, Liesner R, Brock P. Coagulopathy as a presenting feature of Wilms tumour. Eur J Pediatr. 2004;163(7):369-373.

4. Federici AB, Rand JH, Bucciarelli P, Budde U, van Genderen PJ, Mohri H, Meyer D, et al. Acquired von Willebrand syndrome: data from an international registry. Thromb Haemost. 2000;84(2):345-349.

5. Whyte SD, Mark Ansermino J. Anesthetic considerations in the management of Wilms' tumor. Paediatr Anaesth. 2006;16(5):504-513.

6. Palta S, Saroa R, Palta A. Overview of the coagulation system. Indian J Anaesth. 2014;58(5):515-523.

7. Nichols WL, Hultin MB, James AH, Manco-Johnson MJ, Montgomery RR, Ortel TL, Rick ME, et al. von Willebrand disease (VWD): evidence-based diagnosis and management guidelines, the National Heart, Lung, and Blood Institute (NHLBI) Expert Panel report (USA). Haemophilia. 2008;14(2):171-232.

8. Sadler JE. Biochemistry and genetics of von Willebrand factor. Annu Rev Biochem. 1998;67:395-424.

9. Roberts JC, Flood VH. Laboratory diagnosis of von
Willebrand disease. Int J Lab Hematol. 2015;37(Suppl 1):11-17.

10. Bharati KP, Prashanth UR. Von Willebrand disease: an overview. Indian J Pharm Sci. 2011;73(1):7-16.

11. Federici AB. Acquired von Willebrand syndrome: an underdiagnosed and misdiagnosed bleeding complication in patients with lymphoproliferative and myeloproliferative disorders. Semin Hematol. 2006;43(1 Suppl 1):S48-58.

12. Noronha PA, Hruby MA, Maurer HS. Acquired von Willebrand disease in a patient with Wilms tumor. J Pediatr. 1979;95(6):997-999.

13. Fosbury E, Szychot E, Slater O, Mathias M, Sibson K. An 11-year experience of acquired von Willebrand syndrome in children diagnosed with Wilms tumour in a tertiary referral centre. Pediatr Blood Cancer. 2017;64(3):e26246.

14. Vandermeulen EP, Van Aken H, Vermylen J. Anticoagulants and spinal-epidural anesthesia. Anesth Analg. 1994;79(6):1165-1177.

15. Horlocker TT, Wedel DJ, Benzon H, Brown DL, Enneking FK, Heit JA, Mulroy MF, et al. Regional anesthesia in the anticoagulated patient: defining the risks (the second ASRA Consensus Conference on Neuraxial Anesthesia and Anticoagulation). Reg Anesth Pain Med. 2003;28(3):172-197.

16. Beaussier M, El'Ayoubi H, Schiffer E, Rollin M, Parc Y, Mazoit JX, Azizi L, et al. Continuous preperitoneal infusion of ropivacaine provides effective analgesia and accelerates recovery after colorectal surgery: a randomized, double-blind, placebo-controlled study. Anesthesiology. 2007;107(3):461-468.

17. Machoki MS, Millar AJ, Albetyn H, Cox SG, Thomas J, Numanoglu A. Local anesthetic wound infusion versus standard analgesia in paediatric post-operative pain control. Pediatr Surg Int. 2015;31(11):1087-1097.

18. Wang LW, Wong SW, Crowe PJ, Khor KE, Jastrzab G, Parasyn AD, Walsh WR. Wound infusion with local anaesthesia after laparotomy: a randomized controlled trial. ANZ J Surg. 2010;80(11):794-801.

19. Chan SK, Lai PB, Li PT, Wong J, Karmakar MK, Lee KF, Gin T. The analgesic efficacy of continuous wound instillation with ropivacaine after open hepatic surgery. Anaesthesia. 2010;65(12):1180-1186.

20. Forastiere E, Sofra M, Giannarelli D, Fabrizi L, Simone G. Effectiveness of continuous wound infusion of $0.5 \%$ ropivacaine by On-Q pain relief system for postoperative pain management after open nephrectomy. Br J Anaesth. 2008;101(6):841-847.

21. Chalmers DJ, Bielsky A, Wild TT, Siparsky GL, Wilcox DT. Continuous local anesthetic infusion for children with spina bifida undergoing major reconstruction of the lower urinary tract. J Pediatr Urol. 2015;11(2):72 e71-75.

22. Rackelboom T, Le Strat S, Silvera S, Schmitz T, Bassot A, Goffinet F, Ozier Y, et al. Improving continuous wound infusion effectiveness for postoperative analgesia after cesarean delivery: a randomized controlled trial. Obstet Gynecol. 2010;116(4):893-900. 